\title{
Article
}

\section{Pregnancies and Neonatal Outcomes in Patients with Sickle Cell Disease (SCD): Still a (High-)Risk Constellation?}

\author{
Pia Proske 1ㅁ, Laura Distelmaier ${ }^{1,2}$, Carmen Aramayo-Singelmann ${ }^{3}$, Nikolaos Koliastas ${ }^{4}$, \\ Antonella Iannaccone ${ }^{4}$, Maria Papathanasiou ${ }^{5}$, Christian Temme ${ }^{6}$, Hannes Klump ${ }^{6}\left({ }^{0}\right.$, Veronika Lenz ${ }^{6}$, \\ Michael Koldehoff ${ }^{1}$ (D), Alexander Carpinteiro ${ }^{1,7}$, Hans Christian Reinhardt ${ }^{1}$, Angela Köninger 4,8, \\ Alexander Röth ${ }^{1}$, Raina Yamamoto ${ }^{9}$, Ulrich Dührsen ${ }^{1}$ and Ferras Alashkar ${ }^{1, *(D)}$
}

check for updates

Citation: Proske, P.; Distelmaier, L.; Aramayo-Singelmann, C.;

Koliastas, N.; Iannaccone, A.; Papathanasiou, M.; Temme, C.; Klump, H.; Lenz, V.; Koldehoff, M.; et al. Pregnancies and Neonatal Outcomes in Patients with Sickle Cell Disease (SCD): Still a (High-)Risk Constellation? J. Pers. Med. 2021, 11, 870. https://doi.org/10.3390/ jpm11090870

Academic Editors: Adriana Ceci and Petros Kountouris

Received: 29 July 2021

Accepted: 27 August 2021

Published: 30 August 2021

Publisher's Note: MDPI stays neutral with regard to jurisdictional claims in published maps and institutional affiliations.

Copyright: (c) 2021 by the authors. Licensee MDPI, Basel, Switzerland. This article is an open access article distributed under the terms and conditions of the Creative Commons Attribution (CC BY) license (https:/ / creativecommons.org/licenses/by/ $4.0 /)$.
1 Department of Hematology and Stem Cell Transplantation, West German Cancer Center, University Hospital Essen, University of Duisburg-Essen, 45147 Essen, Germany; pia.proske@uk-essen.de (P.P.); laura.distelmaier@vivantes.de (L.D.); michael.koldehoff@uk-essen.de (M.K.); alexander.carpinteiro@uk-essen.de (A.C.); christian.reinhardt@uk-essen.de (H.C.R.); alexander.roeth@uk-essen.de (A.R.); ulrich.duehrsen@uk-essen.de (U.D.)

2 Vivantes, MVZ Neukölln, 12351 Berlin, Germany

3 Department of Pediatrics III, University Children's Hospital Essen, University of Duisburg-Essen, 45147 Essen, Germany; carmen.aramayo-singelmann@uk-essen.de

4 Department of Gynecology and Obstetrics, University of Duisburg-Essen, 45147 Essen, Germany; nikolaos.koliastas@uk-essen.de (N.K.); antonella.iannaccone@uk-essen.de (A.I.); angela.koeninger@barmherzige-regensburg.de (A.K.)

5 Department of Cardiology and Vascular Medicine, West German Heart and Vascular Center, Medical Faculty, University Hospital Essen, 45147 Essen, Germany; maria.papathanasiou@uk-essen.de

6 Institute for Transfusion Medicine, University Hospital Essen, University of Duisburg-Essen, 45147 Essen, Germany; christian.temme@uk-essen.de (C.T.); hannes.klump@uk-essen.de (H.K.); veronika.lenz@uk-essen.de (V.L.)

7 Institute for Molecular Biology, University of Duisburg-Essen, 45147 Essen, Germany

8 Hospital of the Order of St. John of God Regensburg, Clinic for Gynaecology and Obstetrics, 93049 Regensburg, Germany

9 MVZ Dr. Eberhard \& Partner, 44137 Dortmund, Germany; yamamoto@labmed.de

* Correspondence: ferras.alashkar@uk-essen.de

Abstract: Background: This monocentric study conducted at the University Hospital of Essen aims to describe maternal and fetal/neonatal outcomes in sickle cell disease (SCD) documented between 1996 to $2021(\mathrm{~N}=53)$, reflecting the largest monocentric analysis carried out in Germany. Methods/Results: 46 pregnancies in 22 patients were followed. None of the patients died. In total, 35\% (11/31) of pregnancies were preterm. 15 pregnancies in eight patients were conceived on hydroxycarbamide (HC), of which nine had a successful outcome and three were terminated prematurely. There was no difference regarding the rate of spontaneous abortions in patients receiving $\mathrm{HC}$ compared to $\mathrm{HC}$-naive patients prior to conception. In patients other than $\mathrm{HbS} / \mathrm{C}$ disease, pregnancies were complicated by vaso-occlusive crises (VOCs)/acute pain crises (APCs) (96\%, 23/24); acute chest syndrome (ACS) $(13 \%, 3 / 24)$, transfusion demand $(79 \%, 19 / 24)$, urinary tract infections (UTIs) $(42 \%, 10 / 24)$ and thromboembolic events $(8 \%, 2 / 24)$. In $\mathrm{HbS} / \mathrm{C}$ patients complications included: VOCs/APCs (43\%, 3/7; ACS: 14\%, 1/7), transfusion demand $(14 \%, 1 / 7)$, and UTIs $(14 \%, 1 / 7)$. Independent of preterm deliveries, a significant difference with respect to neonatal growth in favor of neonates from $\mathrm{HbS} / \mathrm{C}$ mothers was observed. Conclusion: Our data support the results of previous studies, highlighting the high rate of maternal and fetal/neonatal complications in pregnant SCD patients.

Keywords: pregnancy; sickle cell disease; vaso-occlusive (VOC); transfusion; complications; genotype-phenotype correlation and patient stratification; patient registries and standardization 


\section{Introduction}

Over the last years, sickle cell disease (SCD)-related maternal and neonatal mortality has decreased significantly. This can be ascribed to the availability of evidence-based clinical practice guidelines (CPGs) and preventive measures, antenatal counseling and implementation of newborn screening [1,2]. However, pregnancy in patients with SCD still remains associated with both maternal morbidity and fetal/neonatal morbidity and mortality $[3,4]$. The observation of increased maternal morbidity is supported by the fact that care in SCD throughout pregnancy and in the postpartum period is mainly restricted to supportive measures in addition to transfusion therapy. The reason is that therapeutic modalities require immediate discontinuation due to the fear of possible complications that could particularly endanger fetal well-being [5-7]. Therefore, it is not surprising that sickle cell-associated complications (e.g., anemia, vaso-occlusive crises (VOCs), including acute chest syndrome (ACS), pre-existing cardio-pulmonary and renal complications) often deteriorate in pregnant SCD patients, resulting in frequent inpatient admissions for medical care. In addition, infectious complications, thromboembolic events (TEs), and preeclampsia can further complicate pregnancy and the postpartum period [8].

Erythrocyte sickling may contribute to micro-vascular placental pathology, increasing the risk for decreased placental circulatory blood flow, (acute) fetal hypoxemia, and placental thromboses, all of which are closely associated with a high incidence of perinatal complications. Those can be spontaneous abortions, stillbirths, or an increased rate of preterm deliveries with low gestational weight in neonates. Often, there is an imperative need for cesarean deliveries [8].

The prevalence of SCD and the number of affected children born in Germany is presently unknown. Notwithstanding that reliable epidemiological data are lacking, it is estimated that approximately 3000 to 5000 affected children and adults currently live in Germany. According to conservative estimates, approximately 70 to 150 affected children are born in Germany each year. Furthermore, due to increased immigration within the past years, more children affected by SCD will be born within the next years [9].

Here we report pregnancy outcomes in patients with SCD registered at the Department of Hematology and Stem Cell Transplantation, University Hospital Essen, Germany. To the best of our knowledge, this is the first large German monocentric observational study addressing this clinically important topic in a considerable number of patients.

\section{Materials and Methods}

\subsection{Study Design and Participants}

This is a single-center observational study of pregnancies in women with homozygous SCD (HbSS) or compound heterozygous states, such as $\mathrm{HbS} / \mathrm{C}, \mathrm{HbS} / \beta$-thalassemia, or $\mathrm{HbS} / \mathrm{O}-\mathrm{Arab}$ ( \pm co-inheritance of heterozygous $\alpha$-thalassemia, if tested) who were in part or entirely monitored at the Department of Hematology and Stem Cell Transplantation, University Hospital Essen, Germany, allowing a prospective follow-up. Pregnancies before referral to our department were recorded retrospectively, based on patients' information and medical reports. Data from patients delivering outside of Germany were generally not available. Carrier states for SCD were excluded. The study was approved by the Ethics Committee of the University of Duisburg-Essen and conducted in accordance with the Declaration of Helsinki.

\subsection{Disease-Related Definitions, Methods, and Treatments}

SCD was diagnosed according to international standards at the Hemoglobin Laboratory of the University Hospital Ulm and starting from 2017 at the Medical Care Center Dr. Eberhard and Partner Dortmund via molecular globin gene genetic analyses by polymerase chain reaction (PCR), sequencing and/or multiplex ligation-dependent probe amplification (MLPA). 


\subsection{Sickle Cell-Associated Definitions}

An acute pain crisis (APC) or uncomplicated VOC was defined as an acute onset of pain for which there was no other medical explanation other than vaso-occlusion and which required analgetic treatment (enteral or parenteral), irrespective if the event required hospitalization or was managed at home. ACS or splenic sequestration were referred to as complicated VOCs. ACS was defined based on the finding of a new pulmonary infiltrate in the presence of other signs and symptoms: chest pain, a temperature of $\geq 38.5^{\circ} \mathrm{C}$, tachypnea, wheezing or cough. Splenic sequestration was defined on the basis of left upper quadrant pain, an enlarged spleen, and an acute decrease in hemoglobin $(\mathrm{Hb})$ concentration (e.g., a decrease in $\mathrm{Hb}$ of $2 \mathrm{~g} / \mathrm{dL}$ from baseline).

\subsection{Pregnancy-Related Definitions}

Spontaneous abortion and stillbirth were defined as unsuccessful pregnancy outcomes before or after gestational week (GW) 22. Preterm delivery was defined as delivery before GW 37. Preterm premature rupture of membranes (PPROM) was classified if PROM occurred before GW 37.

\subsection{Treatments}

Treatment with hydroxycarbamide (HC) was recommended according to international guidelines and prescribed, if desired. In all HC-treated patients, therapy was discontinued with establishment of pregnancy due to possible teratogenicity $[10,11]$. Prior to pregnancy, none of the patients were treated with crizanlizumab. In patients allowing for pre-conceptional care (PCC), HC was aimed to be discontinued at least three months before conception under close follow-up, if feasible.

Folic acid (5 mg daily) was routinely supplemented. Iron supplementation was restricted to patients with iron deficiency. Extended red blood cell (RBC) phenotyping, including full Rhesus (C, D, E, c, and e), Kell typing (K, k) and screening for other blood group antigens [Kp(a), Kp(b), Duffy (Fy(a), Fy(b)), Kidd (Jk(a), Jk(b)), M, N, S, s, Lewis $(\mathrm{Le}(\mathrm{a}), \mathrm{Le}(\mathrm{b})), \mathrm{Lu}(\mathrm{a}), \mathrm{Lu}(\mathrm{b})]$ was routinely performed. Partners of patients were encouraged to undertake genetic counseling.

In patients with past pregnancies not yet treated at our center, extended RBC phenotyping was performed on the date of their first visit. RBC transfusions were restricted to patients with symptomatic anemia, a peri- or antenatal $\mathrm{Hb}$ concentration $<7 \mathrm{~g} / \mathrm{dL}$, or in pregnant patients with SCD- or fetal-related complications. A RBC exchange (RCE) was recommended for the treatment of severe VOCs (i.e., severe pain crises and/or ACS). Low-molecular-weight-heparins (LMWHs) were prescribed to patients with prior TEs, in the event of inpatient admission, and were routinely applied in the postpartum period. The duration of thromboprophylaxis was at the discretion of the treating physician, based on the individual risk profile and mode of delivery.

\subsection{Statistical Analysis}

Due to the small sample size, statistical analysis was not carried out except for maternal and neonatal outcomes using paired Student's $t$-test and two-tailed Mann-Whitney U test. Differences between groups were considered to be significant at a $p$ value of $<0.05$. Statistical analyses were performed with GraphPad Prism 6.

\section{Results}

In total, 53 pregnancies in 22 females were recorded ( $\mathrm{HbSS}(15 / 22 ; 68 \%)), \mathrm{HbS} / \beta-$ thalassemia $(2 / 22 ; 9 \%), \mathrm{HbS} / \mathrm{C}(4 / 22 ; 18 \%)$, and $\mathrm{HbS} / \mathrm{O}-\mathrm{Arab}(1 / 22 ; 5 \%) \pm$ co-inheritance of heterozygous $\alpha$-thalassemia) (Table 1). Prior to first presentation, seven gravidities occurred outside Germany, of which five were first-time pregnancies. The median age at first conception was 24 years (range, $14-39 ; \mathrm{N}=22$ ). Four patients had one, six had two, and eleven had $\geq 3$ pregnancies. No twin pregnancies were observed. In two patients the diagnosis of SCD was established after first gestation (Pat. 2 and 10). 
Table 1. Maternal characteristics, including antenatal, peri-, and postpartum complications in SCD patients $(\mathrm{N}=22)$.

\begin{tabular}{|c|c|c|c|c|c|c|c|}
\hline \multirow[b]{2}{*}{ Patient-ID } & \multirow{2}{*}{$\begin{array}{l}\text { Genotype ((Genetic) } \\
\text { Origin) }\end{array}$} & \multirow{2}{*}{$\begin{array}{l}\text { Gravida (G), Para (P); } \\
\text { Age (Years) }\end{array}$} & Before Conception & Gestation Period & \multirow{2}{*}{$\begin{array}{l}\text { Country (Abor- } \\
\text { tion/Stillbirth/Delivery) }\end{array}$} & \multirow{2}{*}{$\begin{array}{l}\text { Peripartum Period } \\
\text { SCD-Associated } \\
\text { Complications }\end{array}$} & \multirow{2}{*}{$\begin{array}{l}\text { Postpartum Period } \\
\text { SCD-Associated } \\
\text { Complications }\end{array}$} \\
\hline & & & $\begin{array}{l}\text { SCD-Associated Complication (HC } \\
\text { Dosage; Alloantibodies) }\end{array}$ & SCD-Associated Complications & & & \\
\hline \multicolumn{8}{|c|}{ Exclusively succesful pregnancy outcomes } \\
\hline \multirow[t]{2}{*}{2} & \multirow[t]{2}{*}{ HbSS (Togo) } & G1, P0; 32 & recurrent APCs & $\begin{array}{l}\text { APCs, transfusion demand for RBCs } \\
\text { (hemolytic/symptomatic anemia; } \\
1 \text { unit) }\end{array}$ & Germany & $\begin{array}{l}\text { CS secondary to birth } \\
\text { arrest in the active-phase } \\
\text { arrest }\end{array}$ & $\begin{array}{l}\text { Transfusion demand for } \\
\text { RBCs ( } 2 \text { units) due to } \\
\text { symptomatic anemia }(\mathrm{Hb} \\
5.6 \mathrm{~g} / \mathrm{dL} \text { ); Wound infection } \\
\text { of the cesarean scar }\end{array}$ \\
\hline & & $\mathrm{G} 2, \mathrm{P} 1 ; 38$ & $\begin{array}{l}\text { recurrent APCs, st. p. } \\
\text { cholecystectomy } \\
\text { (cholecystolithiasis) }\end{array}$ & APCs & Germany & APCs & - \\
\hline \multirow[b]{2}{*}{3} & \multirow[b]{2}{*}{ HbSS (Guinea) } & G1, P0; 14 & - & VOCs/APCs & Guinea & NDA & - \\
\hline & & G2, P1; 20 & $\begin{array}{l}\text { APCs (childhood), st. p. left hip joint } \\
\text { endoprosthesis (left femoral head } \\
\text { necrosis), st. p. cholecystectomy } \\
\text { (cholecystolithiasis); HBV /HDV } \\
\text { infection }\end{array}$ & $\begin{array}{l}\text { VOCs, requiring inpatient } \\
\text { admissions, UTIs }\end{array}$ & Germany & PROM, AIS & - \\
\hline \multirow[t]{2}{*}{4} & \multirow[t]{2}{*}{ HbSS (Turkey) } & $\mathrm{G} 1, \mathrm{P} 0 ; 24$ & \multirow{2}{*}{$\begin{array}{l}\text { recurrent VOCs/APCs; intermittent } \\
\text { transfusion demand for RBCs } \\
\text { (hemolytic/symtpomatic } \\
\text { anemia/VOCs), right femoral head } \\
\text { necrosis, st. p. cholecystectomy } \\
\text { (cholecystolithiasis) }\end{array}$} & $\begin{array}{l}\text { VOCs, including ACS } \\
\text { (RBC-exchange transfusion (GW } \\
\text { 15)), requiring inpatient admissions, } \\
\text { recurrent transfusion demand for } \\
\text { RBCs (hemolytic/symptomatic } \\
\text { anemia), UTIs }\end{array}$ & Germany & AIS (GW 37) & $\begin{array}{l}\text { Transfusion demand for } \\
\text { RBCs ( } 2 \text { units) } \\
\text { (symptomatic anemia (Hb } \\
6.5 \mathrm{~g} / \mathrm{dL}))\end{array}$ \\
\hline & & $\mathrm{G} 2, \mathrm{P} 1 ; 27$ & & $\begin{array}{l}\text { VOCs, including ACS (RCE (GW } \\
29+3) \text { ), requiring inpatient } \\
\text { admissions, transfusion demand for } \\
\text { RBCs (hemolytic/symptomatic } \\
\text { anemia), UTIs }\end{array}$ & Germany & - & - \\
\hline 5 & $\begin{array}{l}\text { HbSS } \\
\text { (Guinea) }\end{array}$ & $\begin{array}{l}\text { G1, P0; } 17 \text { (Germany: } \\
\text { GW 30; refugee status) }\end{array}$ & $\begin{array}{l}\text { recurrent VOCs/APCs, intermittent } \\
\text { transfusion demand for RBCs } \\
\text { (hemolytic/symtpomatic anemia, } \\
\text { VOCs (Guinea)) }\end{array}$ & $\begin{array}{l}\text { GW 30: VOC, requiring inpatient } \\
\text { admission, transfusion demand for } \\
\text { RBCs (hemolytic/symptomatic } \\
\text { anemia), UTI }\end{array}$ & Germany & - & - \\
\hline
\end{tabular}


Table 1. Cont.

\begin{tabular}{|c|c|c|c|c|c|c|c|}
\hline \multirow[b]{2}{*}{ Patient-ID } & \multirow{2}{*}{$\begin{array}{l}\text { Genotype ((Genetic) } \\
\text { Origin) }\end{array}$} & \multirow{2}{*}{$\begin{array}{l}\text { Gravida (G), Para (P); } \\
\text { Age (Years) }\end{array}$} & Before Conception & Gestation Period & \multirow{2}{*}{$\begin{array}{l}\text { Country (Abor- } \\
\text { tion/Stillbirth/Delivery) }\end{array}$} & \multirow{2}{*}{$\begin{array}{l}\text { Peripartum Period } \\
\text { SCD-Associated } \\
\text { Complications }\end{array}$} & \multirow{2}{*}{$\begin{array}{l}\text { Postpartum Period } \\
\text { SCD-Associated } \\
\text { Complications }\end{array}$} \\
\hline & & & $\begin{array}{l}\text { SCD-Associated Complication (HC } \\
\text { Dosag; Alloantibodies) }\end{array}$ & SCD-Associated Complications & & & \\
\hline \multirow[t]{2}{*}{6} & \multirow[t]{2}{*}{$\begin{array}{l}\text { HbSS } \\
\text { (Nigeria) }\end{array}$} & $\begin{array}{l}\text { G1, P0; } 26 \text { (refugee } \\
\text { status) }\end{array}$ & - & - & Germany & $\begin{array}{l}\text { CS secondary to } \\
\text { active-phase arrest, } \\
\text { transfusion demand for } \\
\text { RBCs } \\
\text { (hemolytic/symptomatic } \\
\text { anemia; } 2 \text { units) }\end{array}$ & - \\
\hline & & $\mathrm{G} 2, \mathrm{P} 1 ; 28$ & $\begin{array}{l}\text { splenic sequestration (RBC } \\
\text { transfusion), cholecystolithiasis (HC } \\
15 \mathrm{mg} / \mathrm{kg} \mathrm{BW} \text { ) }\end{array}$ & $\begin{array}{l}\text { VOCs/APCs, requiring inpatient } \\
\text { admissions, transfusion demand for } \\
\text { RBCs (hemolytic/symptomatic } \\
\text { anemia), UTIs }\end{array}$ & Germany & $\begin{array}{l}\text { transfusion demand for } \\
\text { RBCs } \\
\text { (PROM/hemolytic/symptom } \\
\text { anemia; } 2 \text { units) }\end{array}$ & \\
\hline 7 & $\begin{array}{l}\text { HbSS } \\
(\text { Togo })\end{array}$ & $\mathrm{G} 1, \mathrm{P} 0 ; 17$ & $\begin{array}{l}\text { recurrent VOCs/APCs, including } \\
\text { ACS, intermittent transfusion } \\
\text { demand for RBCs } \\
\text { (hemolytic/symptomatic anemia, } \\
\text { VOCs), cholecystolithiasis ( } 27 \\
\mathrm{mg} / \mathrm{kg} \text { BW) }\end{array}$ & $\begin{array}{l}\text { VOCs, requiring inpatient } \\
\text { admissions (GW } 9 \text { and } 27) \text {, } \\
\text { transfusion demand for pRBCs } \\
\text { (hemolytic/symptomatic anemia; } 1 \\
\text { unit (GW 9)), UTI }\end{array}$ & Germany & Cervical insufficiency & - \\
\hline 8 & $\mathrm{HbS} / \beta$-thalassemia (Syria) & $\mathrm{G} 1, \mathrm{P} 0 ; 33$ & $\begin{array}{l}\text { recurrent VOCs/APCs, including } \\
\text { ACS (pulmonary embolism); st. p. } \\
\text { splenectomy (transfusion demand } \\
\text { for pRBCs, hemolytic/symptomatic } \\
\text { anemia, VOCs (Syria)), } \\
\text { cholecystolithiasis (HC: } 20 \mathrm{mg} / \mathrm{kg} \\
\text { BW) }\end{array}$ & $\begin{array}{l}\text { Transfusion demand for RBCs } \\
\text { (hemolytic/symptomatic anemia; } \\
\mathrm{Hb}<7 \mathrm{~g} / \mathrm{dL} ; 5 \text { units, GW 20, 25, } 30 \\
\text { (borderline doppler indices; } \\
\text { intrauterine growth restriction), } \\
\text { APC }\end{array}$ & Germany & $\begin{array}{l}\text { CS (secondary to } \\
\text { pathologic CTG and } \\
\text { doppler indices, placental } \\
\text { insufficiency) }\end{array}$ & $\begin{array}{l}\text { Transfusion demand for } \\
\text { RBCs ( } 2 \text { units) due to } \\
\text { symptomatic anemia ( } \mathrm{Hb} \\
8.5 \mathrm{~g} / \mathrm{dL} \text { ) }\end{array}$ \\
\hline \multirow{3}{*}{9} & \multirow{3}{*}{$\begin{array}{l}\mathrm{HbS} / \mathrm{C} \\
\text { (Ghana) }\end{array}$} & $\mathrm{G} 1, \mathrm{P} 0 ; 30$ & - & - & Germany & - & - \\
\hline & & $\mathrm{G} 2, \mathrm{P} 1 ; 31$ & - & - & Germany & - & - \\
\hline & & $\mathrm{G} 3, \mathrm{P} 2 ; 32$ & - & - & Germany & AIS & - \\
\hline 10 & $\begin{array}{l}\text { HbS/C } \\
\text { (Ghana) }\end{array}$ & $\mathrm{G} 1, \mathrm{P} 0 ; 35$ & APCs & $\mathrm{APCs}$ & Germany & - & - \\
\hline \multicolumn{8}{|c|}{ Successful and unsuccessful pregnancy outcomes } \\
\hline \multirow[b]{3}{*}{11} & \multirow{3}{*}{$\begin{array}{l}\text { HbSS- } \alpha^{+} \text {-thalassemia } \\
\text { (Angola); } \\
\text { Delta-storage pool disease } \\
\text { (Diagnosis: } 4 \text { th pregnancy) }\end{array}$} & $\mathrm{G} 1, \mathrm{P} 0 ; 20$ & \multirow{3}{*}{$\begin{array}{l}\text { VOCs/APCs, intermittent } \\
\text { transfusion demand for RBCs } \\
\text { (hemolytic/symptomatic anemia, } \\
\text { VOCs) (childhood), } \\
\text { cholecystolithiasis, st. p. } \\
\text { splenectomy }\end{array}$} & - & \multicolumn{3}{|c|}{ Germany (spontaneous abortion, GW 6) } \\
\hline & & $\mathrm{G} 2, \mathrm{P} 0 ; 21$ & & - & \multicolumn{3}{|c|}{ Germany (spontaneous abortion, GW 11) } \\
\hline & & $\mathrm{G} 3, \mathrm{P} 0 ; 22$ & & $\begin{array}{l}\text { VOCs/APCs, transfusion demand } \\
\text { for RBCs (1 unit) }\end{array}$ & Germany & $\begin{array}{l}\text { VOC, transfusion demand } \\
\text { for RBCs } \\
\text { (hemolytic/symptomatic } \\
\text { anemia; } 1 \text { unit) }\end{array}$ & - \\
\hline
\end{tabular}


Table 1. Cont.

\begin{tabular}{|c|c|c|c|c|c|c|c|}
\hline \multirow[b]{2}{*}{ Patient-ID } & \multirow{2}{*}{$\begin{array}{l}\text { Genotype ((Genetic) } \\
\text { Origin) }\end{array}$} & \multirow{2}{*}{$\begin{array}{l}\text { Gravida (G), Para (P); } \\
\text { Age (Years) }\end{array}$} & Before Conception & Gestation Period & \multirow{2}{*}{$\begin{array}{l}\text { Country (Abor- } \\
\text { tion/Stillbirth/Delivery) }\end{array}$} & \multirow{2}{*}{$\begin{array}{l}\text { Peripartum Period } \\
\text { SCD-Associated } \\
\text { Complications }\end{array}$} & \multirow{2}{*}{$\begin{array}{l}\text { Postpartum Period } \\
\text { SCD-Associated } \\
\text { Complications }\end{array}$} \\
\hline & & & $\begin{array}{l}\text { SCD-Associated Complication (HC } \\
\text { Dosage; Alloantibodies) }\end{array}$ & SCD-Associated Complications & & & \\
\hline & & $\mathrm{G} 4, \mathrm{P} 1 ; 25$ & & $\begin{array}{l}\text { VOCs/APCs, requiring inpatient } \\
\text { admission (GW 26, 28), transfusion } \\
\text { demand for RBCs } \\
\text { (hemolytic/symptomatic anemia; } 6 \\
\text { units); UTI-> urosepsis (GW 28) }\end{array}$ & Germany & $\begin{array}{l}\text { CS (fetal subdural hemat } \\
\text { (hematoma evacuation); } \\
\text { re-re-re-sectio (hysterecto } \\
\text { (hematoma evacuation); } \\
\text { (post-bleeding); hemorrh } \\
\text { (pulmonary embolism); } \\
\text { (overall: } 49 \text { pRBC units) } \\
\text { postpartum cardiomyopa }\end{array}$ & $\begin{array}{l}\text { GW } 29+5) \text {; re-sectio } \\
\text { sectio (post-bleeding); } \\
\text { re-re-re-re-sectio } \\
\text {-re-re-re-sectio } \\
\text { shock; DIC; ACS } \\
\text { usion demand for RBCs } \\
\text { latetes (16 units); } \\
\text { (HFrEF; EF 20-35\%) }\end{array}$ \\
\hline \multirow[t]{2}{*}{12} & \multirow{2}{*}{$\begin{array}{l}\text { HbSS- } \alpha^{+} \text {-thalassemia } \\
\text { (Nigeria) }\end{array}$} & $\mathrm{G} 2, \mathrm{P} 1 ; 30$ & \multirow[t]{2}{*}{ VOCs/APCs } & APCs & Germany (elective abortion) & & \\
\hline & & $\mathrm{G} 2, \mathrm{P} 1 ; 33$ & & APCs & Germany (spontaneous aborti & & \\
\hline \multirow{3}{*}{13} & \multirow{3}{*}{$\begin{array}{l}\text { HbSS- } \alpha^{+} \text {-thalassemia } \\
\text { (Zaire) }\end{array}$} & G1, P0; 17 & $\begin{array}{l}\text { VOCs/APCs, transfusion demand } \\
\text { for RBCs (hemolytic symptomatic } \\
\text { anemia, VOCs); st. p. splenectomy; } \\
\text { st. p. cholecystectomy } \\
\text { (cholecystolithiasis), infections (HC: } \\
35 \mathrm{mg} / \mathrm{kg} \mathrm{BW} \text { ) }\end{array}$ & - & Germany (elective abortion) & & \\
\hline & & G2, P0; 19 & $\begin{array}{l}\text { VOCs/APCs, transfusion demand } \\
\text { for RBCs (hemolytic symptomatic } \\
\text { anemia, VOCs), left DVT (HC: } 25 \\
\text { mg/kg BW) }\end{array}$ & $\begin{array}{l}\text { VOCs/APCs, requiring inpatient } \\
\text { admissions (GW 16, 32), transfusion } \\
\text { demand for pRBCs } \\
\text { (hemolytic/symptomatic anemia; } 6 \\
\text { units), febrile UTIs }\end{array}$ & Germany & $\begin{array}{l}\text { PROM, CS secondary to } \\
\text { active-phase arrest }\end{array}$ & $\begin{array}{l}\text { Transfusion demand for } \\
\text { RBCs } \\
\text { (hemolytic/symptomatic } \\
\text { anemia; } 1 \text { unit) }\end{array}$ \\
\hline & & G3, P1; 22 & $\begin{array}{l}\text { VOCs/APCs, including ACSs (3rd } \\
\text { ACS: vvECMO), transfusion } \\
\text { demand for RBCs } \\
\text { (hemolytic/symptomatic anemia, } \\
\text { VOCs), gram-negative port infection } \\
\text { (HC: } 25 \mathrm{mg} / \mathrm{kg} \text { BW) }\end{array}$ & $\begin{array}{l}\text { VOCs/APCs, requiring inpatient } \\
\text { admissions (GW } 8,11,24,29,33) \text {, } \\
\text { transfusion demand for RBCs } \\
\text { (hemolytic/symptomatic anemia; } 4 \\
\text { units) }\end{array}$ & Germany & PROM & - \\
\hline \multirow{4}{*}{14} & \multirow{4}{*}{$\begin{array}{l}\text { HbSS } \\
\text { (Dominican Republic) }\end{array}$} & G1, P0; 18 & \multirow{4}{*}{$\begin{array}{l}\text { VOCs/APCs, including ACS, } \\
\text { intermittent transfusion demand for } \\
\text { RBCs (hemolytic/symptomatic } \\
\text { anemia, VOCs), infections, } \\
\text { cholecystolithiasis }\end{array}$} & $\begin{array}{l}\text { VOCs/APCs, transfusion demand } \\
\text { for RBCs (hemolytic/symptomatic } \\
\text { anemia; } 3 \text { units) }\end{array}$ & Dominican Republic & - & - \\
\hline & & G2, P1; 21 & & - & Dominican Republic (spontan & eous abortion) & \\
\hline & & G3, P1; 25 & & $\begin{array}{l}\text { VOCs/APCs, transfusion demand } \\
\text { for RBCs (hemolytic/symptomatic } \\
\text { anemia; } 2 \text { units) }\end{array}$ & Dominican Republic & - & - \\
\hline & & G4, P2; 30 & & - & Germany (spontaneous aborti & & \\
\hline
\end{tabular}


Table 1. Cont.

\begin{tabular}{|c|c|c|c|c|c|c|c|}
\hline \multirow[b]{2}{*}{ Patient-ID } & \multirow{2}{*}{$\begin{array}{l}\text { Genotype ((Genetic) } \\
\text { Origin) }\end{array}$} & \multirow{2}{*}{$\begin{array}{l}\text { Gravida (G), Para (P); } \\
\text { Age (Years) }\end{array}$} & Before Conception & Gestation Period & \multirow{2}{*}{$\begin{array}{l}\text { Country (Abor- } \\
\text { tion/Stillbirth/Delivery) }\end{array}$} & Peripartum Period & Postpartum Period \\
\hline & & & $\begin{array}{l}\text { SCD-Associated Complication (HC } \\
\text { Dosage; Alloantibodies) }\end{array}$ & SCD-Associated Complications & & $\begin{array}{l}\text { SCD-Associated } \\
\text { Complications }\end{array}$ & $\begin{array}{l}\text { SCD-Associated } \\
\text { Complications }\end{array}$ \\
\hline \multirow{3}{*}{15} & \multirow{3}{*}{$\begin{array}{l}\text { HbSS } \\
\text { (Congo) }\end{array}$} & $\mathrm{G} 1, \mathrm{P} 0 ; 24$ & $\begin{array}{l}\text { VOCs/APCs, including ACS; } 4 \text { yr. } \\
\text { history of transfusion therapy } \\
\text { (20-24th years of age; Italy) }\end{array}$ & $\begin{array}{l}\text { VOCs/APCs, requiring inpatient } \\
\text { admissions (SP(E)BT, every } 3 \text { weeks) }\end{array}$ & Italy & - & $\begin{array}{l}\text { Continuation of } \\
\text { transfusion therapy }\end{array}$ \\
\hline & & $\mathrm{G} 2, \mathrm{P} 1 ; 29$ & VOCs/APCs (HC: 20 mg/kg BW) & $\begin{array}{l}\text { VOCs, requiring inpatient } \\
\text { admissions, transfusion demand for } \\
\text { RBCs (hemolytic/symptomatic } \\
\text { anemia; } 3 \text { units) }\end{array}$ & Germany & HELLP-Syndrome & - \\
\hline & & $\mathrm{G} 3, \mathrm{P} 2 ; 32$ & VOCs/APCs (HC: 20 mg/kg BW) & - & \multicolumn{2}{|l|}{ Germany (spontaneous abortion) } & \\
\hline \multirow{3}{*}{16} & \multirow{3}{*}{$\begin{array}{l}\text { HbSS } \\
\text { (Angola) }\end{array}$} & G1, P0; 20 & \multirow[b]{2}{*}{$\begin{array}{l}\text { VOCs/APCs, transfusion demand } \\
\text { for RBCs (hemolytic/symptomatic } \\
\text { anemia) (Angola/Belgium) (HC: } \\
15 \mathrm{mg} / \mathrm{kg} \text { BW; alloantibodies: } \\
\text { anti- E, -M) }\end{array}$} & - & \multicolumn{2}{|l|}{ Belgium (sponatenous abortion) } & \\
\hline & & $\begin{array}{l}\text { G2, P1; } 22 \text { (Germany: } \\
\text { GW 29, refugee status) }\end{array}$ & & $\begin{array}{l}\text { VOCs/APCs, requiring inpatient } \\
\text { admissions (GW 29), transfusion } \\
\text { demand for RBCs } \\
\text { (hemolytic/symptomatic anemia; } 2 \\
\text { units), possible DHTR (Anti-S) } \\
\text { following RBC transfusion in the } \\
\text { context of a VOC (DD febrile } \\
\text { hyperhemolytic VOC (UTI)) (re-start } \\
\text { HU ( } 20 \mathrm{mg} / \mathrm{kg} \text { BW), GW } 34+\text { EPO) }\end{array}$ & Germany & $\begin{array}{l}\text { vaginal bleedings (GW 35), } \\
\text { PPROM }\end{array}$ & \\
\hline & & G3, P2; 24 & $\begin{array}{l}\text { VOCs/APCs, including ACS, } \\
\text { transfusion demand for RBCs } \\
\text { (hemolytic/symptomatic anemia) } \\
\text { (HC: } 20 \mathrm{mg} / \mathrm{kg} \text { BW + EPO; } \\
\text { alloantibodies: anti- E, -M, -S) }\end{array}$ & $\begin{array}{l}\text { VOCs/APCs, requiring inpatient } \\
\text { admissions (GW } 13 \text { and 17) } \\
\text { (infectious-related), transfusion } \\
\text { demand for RBCs } \\
\text { (hemolytic/symptomatic anemia; } 5 \\
\text { units) (EPO throughout pregnancy; } \\
\text { re-start HU GW 30; } 15 \mathrm{mg} / \mathrm{kg} \mathrm{BW} \text { ) }\end{array}$ & Germany & - & - \\
\hline \multirow{2}{*}{17} & \multirow{2}{*}{$\begin{array}{l}\mathrm{HbS} / \mathrm{C}-\alpha^{+} \text {-thalassemia } \\
\text { (Ghana) }\end{array}$} & G1, P0; 17 & \multirow{2}{*}{ VOCs/APCs } & - & \multicolumn{2}{|l|}{ Germany (elective abortion) } & \\
\hline & & $\mathrm{G} 2, \mathrm{P} 0 ; 22$ & & - & Germany & - & - \\
\hline \multirow{3}{*}{18} & \multirow{3}{*}{$\begin{array}{l}\mathrm{HbS} / \mathrm{C} \\
\text { (Nigeria) }\end{array}$} & G1, P0; 24 & \multirow{3}{*}{ VOCs/APCs } & - & Germany (elective abortion) & & \\
\hline & & $\mathrm{G} 2, \mathrm{P} 0 ; 26$ & & $\begin{array}{l}\text { APCs, transfusion demand for RBCs } \\
\text { (hemolytic/symptomatic anemia; } \\
2 \text { units) }\end{array}$ & Germany & - & APCs \\
\hline & & G3, P1; 28 & & APCs & Germany & $\begin{array}{l}\text { VOC-infectious related } \\
\text { (UTI -> pyelonephritis) -> } \\
\text { ACS }\end{array}$ & - \\
\hline \multirow{3}{*}{19} & \multirow{3}{*}{$\begin{array}{l}\text { HbS/O-Ara } \\
\text { (Kenya) }\end{array}$} & G1, P0; 28 & \multirow{3}{*}{$\begin{array}{l}\text { recurrent VOCs (child- and } \\
\text { adulthood), including recurrent } \\
\text { ACSs (childhood) and transfusion } \\
\text { demand for RBCs } \\
\text { (symptomatic/hemolytic anemia, } \\
\text { VOCs) }\end{array}$} & DVT & \multicolumn{2}{|l|}{ Germany (stillbirth, GW 26) } & \\
\hline & & $\mathrm{G} 2, \mathrm{P} 0 ; 28$ & & - & \multicolumn{2}{|c|}{ Germany (spontaneous abortion, GW 11) } & \\
\hline & & G3, P0; 29 & & VOCs & Germany & Placental abruption & - \\
\hline
\end{tabular}


Table 1. Cont

\begin{tabular}{|c|c|c|c|c|c|c|c|}
\hline \multirow[b]{2}{*}{ Patient-ID } & \multirow{2}{*}{$\begin{array}{l}\text { Genotype ((Genetic) } \\
\text { Origin) }\end{array}$} & \multirow{2}{*}{$\begin{array}{l}\text { Gravida (G), Para (P); } \\
\text { Age (Years) }\end{array}$} & Before Conception & Gestation Period & \multirow{2}{*}{$\begin{array}{l}\text { Country (Abor- } \\
\text { tion/Stillbirth/Delivery) }\end{array}$} & \multirow{2}{*}{$\begin{array}{l}\text { Peripartum Period } \\
\text { SCD-Associated } \\
\text { Complications }\end{array}$} & \multirow{2}{*}{$\begin{array}{l}\text { Postpartum Perioc } \\
\text { SCD-Associated } \\
\text { Complications }\end{array}$} \\
\hline & & & $\begin{array}{l}\text { SCD-Associated Complication (HC } \\
\text { Dosage; Alloantibodies) }\end{array}$ & SCD-Associated Complications & & & \\
\hline \multicolumn{8}{|c|}{ Unsuccessful pregnancy outcomes } \\
\hline \multirow{3}{*}{20} & \multirow{3}{*}{$\begin{array}{l}\text { HbSS- } \alpha^{+} \text {-thalassemia } \\
\text { (Suriname) }\end{array}$} & $\mathrm{G} 1, \mathrm{P} 0 ; 31$ & VOCs/APCs (childhood) & - & \multicolumn{3}{|c|}{ Germany (ectopic pregnancy (IVF); medical abortion) } \\
\hline & & G3, P0; 32 & - & - & \multicolumn{3}{|c|}{ Germany (spontaneous abortion, (IVF)) } \\
\hline & & $\mathrm{G} 3, \mathrm{P} 0 ; 32$ & - & - & \multicolumn{3}{|c|}{ Germany (ectopic pregnancy (IVF); medical abortion) } \\
\hline \multirow{4}{*}{21} & \multirow{4}{*}{$\begin{array}{l}\text { HbSS } \\
\text { (Congo) }\end{array}$} & G1, P0; 22 & $\begin{array}{l}\text { Recurrent VOCs/APCs, including } \\
\text { ACS, intermittent transfusion } \\
\text { demand for RBCs } \\
\text { (hemolytic/symptomatic anemia, } \\
\text { VOCs; 8 units), right femoral head } \\
\text { necrosis }\end{array}$ & - & Germany (elective abortic & & \\
\hline & & $\mathrm{G} 2, \mathrm{P} 0 ; 24$ & $\begin{array}{l}\text { recurrent VOCs/APCs, intermittent } \\
\text { transfusion demand for RBCs } \\
\text { (hemolytic/symptomatic anemia; } \\
2 \text { units), st. p. right hip joint } \\
\text { endoprothesis (right femoral head } \\
\text { necrosis), left humerus head } \\
\text { necrosis (HC: } 16 \mathrm{mg} / \mathrm{kg} \mathrm{BW} \text { ) }\end{array}$ & - & \multicolumn{3}{|l|}{ Germany (elective abortion) } \\
\hline & & G3, P0; 28 & $\begin{array}{l}\text { recurrent VOCs/APCs, including } \\
\text { ACS, intermittent transfusion } \\
\text { demand for RBCs } \\
\text { (hemolytic/symptomatic anemia; } \\
2 \text { units) (HC: } 21 \mathrm{mg} / \mathrm{kg} \mathrm{BW} \text { ) }\end{array}$ & - & \multicolumn{3}{|c|}{ Germany (elective abortion) } \\
\hline & & $\mathrm{G} 4, \mathrm{P} 0 ; 28$ & $\begin{array}{l}\text { recurrent VOCs/APCs (HC: } \\
25 \mathrm{mg} / \mathrm{kg} \mathrm{BW} \text { ) }\end{array}$ & - & \multicolumn{3}{|c|}{ Germany (spontaneous abortion) } \\
\hline \multirow[t]{2}{*}{22} & \multirow[t]{2}{*}{$\begin{array}{l}\text { HbS- } \beta \text {-thalassemia } \\
\text { (Cuba) }\end{array}$} & G1, P0; 18 & $\begin{array}{l}\text { recurrent VOCs/APCs, including } \\
\text { ACS, st. p. splenectomy (splenic } \\
\text { sequestration), transfusion demand } \\
\text { for RBCs (hemolytic/symptomatic } \\
\text { anemia, VOCs) }\end{array}$ & - & \multicolumn{3}{|c|}{ Germany (elective abortion) } \\
\hline & & $\mathrm{G} 2, \mathrm{P} 0 ; 39$ & $\begin{array}{l}\text { recurrent VOCs/APCs, including } \\
\text { ACS, bi-femoral head necrosis (st. p. } \\
\text { femoral head cannulation) }\end{array}$ & - & \multicolumn{3}{|c|}{ Germany (elective abortion) } \\
\hline
\end{tabular}

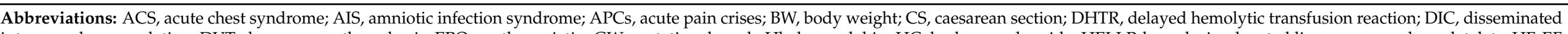

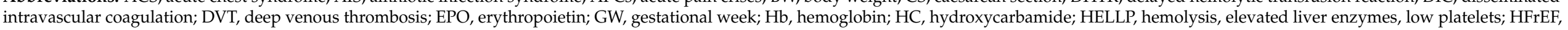

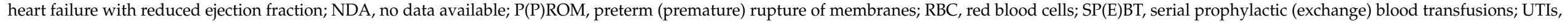
urinary tract infections; VOCs, vaso-occlusive crises. 
Fifteen conceptions occurred in eight of the 22 patients under HC treatment (median maximum HC dosage: $20 \mathrm{mg} / \mathrm{kg}$ body weight (BW) (range, 8-35)), with three documented pregnancies in three patients and two in one patient. Antenatal counseling allowing for adequate time of $\mathrm{HC}$ discontinuation was possible in only one patient (Pat. 8).

At first presentation, alloimmunization to RBC antigens was observed in two patients (Pat. 1: anti-K and 16: anti-M, and -E). Both of which had a medical history of repeated RBC transfusions.

\subsection{Maternal, Fetal, and Neonatal Outcomes}

None of the patients died. In total, 31 of the $53(58 \%)$ documented pregnancies in 22 patients had a successful outcome (median age at conception: 26 years, range 14-39) with 26 deliveries observed in Germany (Table 2). Excluding elective $(\mathrm{N}=9)$ or medicalinduced abortions $(\mathrm{N}=2), 74 \%(31 / 42)$ of pregnancies were successful. Ten pregnancies in eight patients resulted in spontaneous abortions, including one patient requiring in-vitro fertilization (IVF) (28 years of age (median), range 20-33).

Overall, patient age was independent with respect to pregnancy outcome (successful versus unsuccessful), irrespective (prior) HC exposure: overall: $p=0.7477$; severe genotypes: $p=0.4697$. Preterm deliveries $(35 \%(11 / 31))$ were secondary to premature rupture of membranes (PROM), HELLP syndrome (hemolysis, elevated liver enzymes, low platelets), placenta insufficiency or abruption, pathologic fetal cardiotocographs (CTGs) and doppler indices, amniotic infection syndrome (AIS), maternal urosepsis, fetal subdural hematoma, spontaneous onset of labor in a multigravida patient, or due to a combination of two causes. Medical data concerning the reasons for preterm deliveries in patients giving birth outside Germany were in general not available.

In HC-pre-treated patients, nine of the 15 documented pregnancies had a successful outcome. Three pregnancies were terminated prematurely by elective abortions and three pregnancies resulted in spontaneous abortions, compared to six spontaneous abortions of the 22 pregnancies in patients with no prior exposure to $\mathrm{HC}$. None of the patients with $\mathrm{HbS} / \mathrm{C}$ disease experienced spontaneous abortions/stillbirths or received $\mathrm{HC}$ prior to pregnancy (median age at conception: 28 years, range 17-35).

The rate of spontaneous abortions in patients with severe genotypes, if excluding the one patient requiring IVF (independent risk factor), was independent of prior $\mathrm{HC}$ treatment (HC-treated: 25\% (3/12); HC-naive: 27\% (6/22)). Of note, two pregnancies in one patient were conceived under HC (Pat. 16).

The histological evaluation of the placenta from the one patient who experienced a stillbirth was not available. In patients suffering from recurrent $(\geq 2)$ miscarriages $(\mathrm{N}=3)$, only one (Pat. 19) was tested for other causes of loss of pregnancy. In another patient (Pat. 11), the diagnosis of delta-storage pool disease was confirmed during her fourth pregnancy.

\subsection{Gestational-, Peri-, and Postpartum Phase in Patients with Severe Genotypes (HbSS, $\mathrm{HbS} / \beta$-Thalassemia, and HbS/O-Arab Disease)}

In patients with severe genotypes and successful pregnancies $(\mathrm{N}=24)$, APCs or VOCs were described by all patients during gestation, except for one carrying the HbSS genotype $(96 \%, 23 / 24)$. These events predominately occurred during the second and third trimester, requiring in part (recurrent) inpatient admissions (54\%, (13/24)) for (parenteral) analgetic treatment, and/or RBC transfusion support secondary to VOCs, or because of (infectiousrelated) hemolytic and/or symptomatic anemia. Throughout the observation time, two complicated VOCs (ACS) were observed in one HC-naive HbSS patient (Pat. 4) during her two successive pregnancies (GW 15 and $29+3$ ) necessitating manual RCE. An additional ACS was observed in the postpartum period in a second HbSS patient (Pat. 11) (ACS rate in patients with severe disease-associated genotypes: $13 \%(3 / 24))$. 
Table 2. Delivery and neonatal status in women with SCD $(\mathrm{N}=31)$.

\begin{tabular}{|c|c|c|c|c|c|c|c|c|c|c|c|}
\hline \multirow{2}{*}{$\begin{array}{l}\text { Patient-ID/Genotype } \\
\text { (Mother) }\end{array}$} & \multirow[b]{2}{*}{ Pregnancy } & \multicolumn{3}{|c|}{ Delivery } & \multicolumn{7}{|c|}{ Newborn } \\
\hline & & Time $^{a}$ & Type & $\begin{array}{c}\text { Country } \\
\text { (Birth) }\end{array}$ & Apgar Score ${ }^{b}$ & $\mathrm{ApH}$ & $\mathrm{VpH}$ & $\mathrm{BE}(\mathrm{mmol} / \mathrm{l})$ & Weight (g) & Height (cm) & $\begin{array}{c}\text { Head } \\
\text { Circumference (cm) }\end{array}$ \\
\hline \multicolumn{12}{|c|}{ HbSS ( $\pm \alpha^{+}$-thalassemia) } \\
\hline 1 & 1 st & $38+4$ & CS & Germany & $9 / 10 / 10$ & 7.25 & 7.3 & -1.2 & 3090 & 50 & 34 \\
\hline \multirow{2}{*}{2} & 1st & $39+1$ & CS & Germany & $8 / 10 / 10$ & 7.16 & - & -11.5 & 2620 & 48 & 33 \\
\hline & 2nd & $38+1$ & CS & Germany & $9 / 10 / 10$ & 7.25 & - & -6.7 & 2730 & 50 & 33.5 \\
\hline \multirow{2}{*}{3} & 1st & 36 & CS & Guinea & - & - & - & - & - & - & - \\
\hline & 2nd & $37+1$ & VD & Germany & $9 / 10 / 10$ & 7.2 & - & - & 2710 & 48 & 32 \\
\hline \multirow{2}{*}{4} & $1 \mathrm{st}$ & $37+5$ & CS & Germany & $9 / 10 / 10$ & 7.32 & 7.38 & - & 2610 & 47 & 34.5 \\
\hline & 2nd & $39+5$ & VD & Germany & $9 / 10 / 10$ & 7.29 & 7.36 & - & 2770 & 46 & 33.5 \\
\hline 5 & $1 \mathrm{st}$ & $37+5$ & VD & Germany & $6 / 7 / 8$ & 7.19 & - & -10 & 1980 & 46 & 31 \\
\hline \multirow{2}{*}{6} & $1 \mathrm{st}$ & $38+3$ & CS & Germany & $7 / 9 / 10$ & 7.29 & - & -5.8 & 2570 & 47 & 33 \\
\hline & 2nd & $35+1$ & CS & Germany & $7 / 8 / 9$ & 7.34 & 7.37 & - & 1900 & 40 & 31 \\
\hline 7 & 1 st & $37+1$ & VD & Germany & $9 / 10 / 10$ & 7.10 & 7.27 & 11 & 2185 & 46 & 32 \\
\hline 11 & 4th & $29+5$ & CS & Germany & $7 / 8 / 8$ & 7.37 & - & - & 1600 & 41 & 29 \\
\hline 12 & $1 \mathrm{st}$ & 36 & CS & Nigeria & - & - & - & - & - & - & - \\
\hline \multirow{2}{*}{13} & 2 nd & $38+6$ & CS & Germany & $9 / 10 / 10$ & 7.32 & 7.37 & -2.4 & 2845 & 47 & 33 \\
\hline & 3rd & $37+7$ & VD & Germany & $9 / 10 / 10$ & 7.29 & 7.31 & - & 2645 & 50 & 34 \\
\hline \multirow{2}{*}{14} & $1 \mathrm{st}$ & 39 & VD & $\begin{array}{c}\text { Dominican } \\
\text { Republic }\end{array}$ & - & - & - & - & - & - & - \\
\hline & $3 \mathrm{rd}$ & 40 & CS & $\begin{array}{c}\text { Dominican } \\
\text { Republic }\end{array}$ & - & - & - & - & - & - & - \\
\hline \multirow{2}{*}{15} & $1 \mathrm{st}$ & 32 & CS & Italy & - & - & - & - & - & - & - \\
\hline & 2nd & $34+1$ & CS & Germany & $8 / 8 / 9$ & 7.3 & 7.37 & & 2320 & - & - \\
\hline \multirow{2}{*}{16} & 2nd & $35+2$ & VD & Germany & $10 / 10 / 10$ & 7.27 & 7.28 & -5.6 & 2540 & 46 & 32 \\
\hline & $3 r d$ & $34+4$ & VD & Germany & $7 / 8 / 9$ & 7.22 & 7.26 & -4.5 & 2300 & 42 & 31 \\
\hline
\end{tabular}


Table 2. Cont.

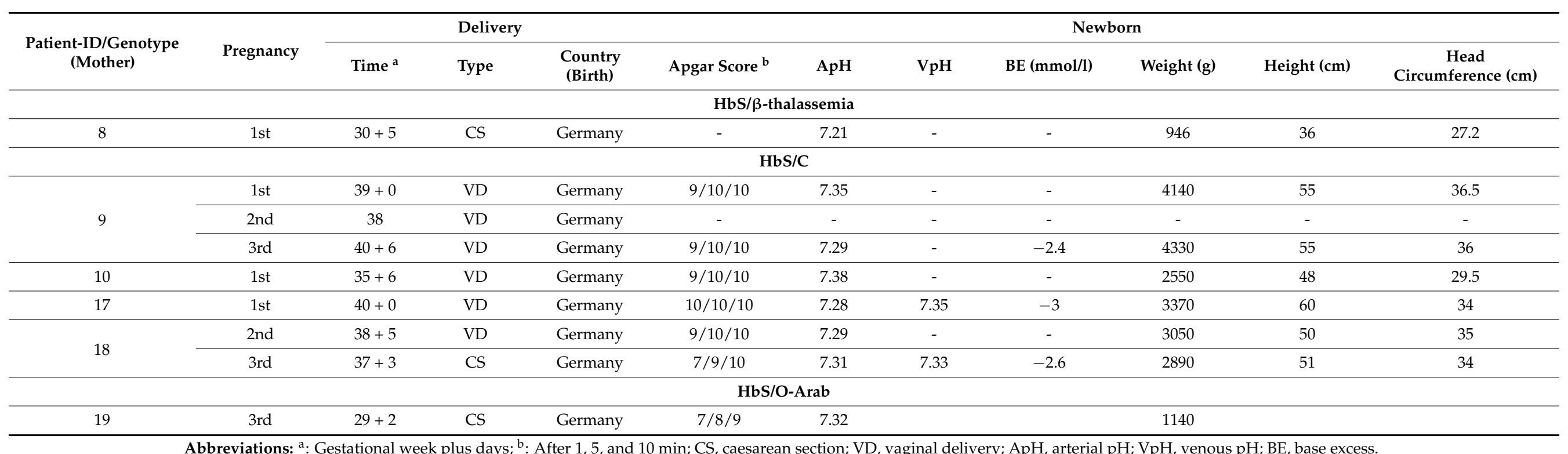

Abbreviations: ${ }^{\text {a }}$ Gestational week plus days; ${ }^{\text {b }: ~ A f t e r ~ 1, ~ 5, ~ a n d ~} 10$ min; $\mathrm{CS}$, caesarean section; $\mathrm{VD}$, vaginal delivery; $\mathrm{ApH}$, arterial $\mathrm{pH}$; $\mathrm{VpH}$, venous pH; $\mathrm{BE}$, base excess. 
RBC transfusions were necessary in 75\% (18/24) of the patients. One HC-naive patient treated in Italy presenting with recurrent VOCs (including ACS) prior to pregnancy, received serial prophylactic (exchange) blood transfusions (SP(E)BTs) for four years which were continued throughout pregnancy and thereafter every three weeks (Pat. 15). In another pre-conceptionally HC-treated patient (Pat. 8), SPBTs (every three weeks, starting from GW 20) were indicated due to suspicious doppler indices and symptomatic/hemolytic anemia.

One possible delayed hemolytic transfusion reaction (DHTR) with diagnosis of an alloantibody against the S-antigen (DD hyper-hemolytic VOC) despite transfusion of extended-phenotyped and matched RBCs was observed in one HbSS patient (Pat. 16, GW 29) already expressing irregular antibodies against erythrocytic antigens (anti-E, antiM). To avoid further RBC transfusions, resumption of $\mathrm{HC}(20 \mathrm{mg} / \mathrm{kg} \mathrm{BW})$ in combination with erythropoietin (EPO) in GW 34 prior to delivery in GW $35+2$ was indicated. Due to transfusion-dependent anemia in her subsequent pregnancy despite continuation of EPO, resumption of HC (15 mg/kg BW; GW 30) was mandatory prior to delivery $(\mathrm{GW} 34+4)$. In the presence of $\mathrm{HC}$, breastfeeding was avoided in this patient.

Overall, thromboembolic complications were seen in two patients (left DVT (Pat. 19; GW 26) and pulmonary embolism in the context of ACS during puerperium (Pat. 11)). Infectious complications were restricted to (recurrent) urinary tract infections (UTIs) and documented in ten out of the 24 successful pregnancies $(42 \%)$, including maternal urosepsis in third trimester (GW 28) in one patient (Pat. 11).

The peripartum complications included $\mathrm{P}(\mathrm{P}) \mathrm{ROM}$ in five pregnancies, possibly associated with or occurred independently to placental abruption, cervical insufficiency, or amniotic infection syndrome (AIS). One patient suffered from HELLP syndrome. VOCs/APCs and/or RBC transfusion demand in the peripartum period were observed in five of the pregnancies (21\%). A caesarean section (CS) secondary to pregnancy-, including activephase arrest and/or pathologic doppler indices $(\mathrm{N}=4)$, or SCD-related complications was mandatory in $42 \%(10 / 24)$ of patients for whom medical data were available, resulting in an overall CS rate of $67 \%(16 / 24)$. In the postpartum period, RBC transfusions were required in four patients. Postpartum cardiomyopathy was seen in one patient (Pat. 11).

Following delivery, HC was re-initiated, if breastfeeding was not desired. In breasfeeding patients, treatment was re-initiated as soon as weaning was ensured.

\subsection{Gestational-, Peri-, and Postpartum Phase in Patients with HbS/C Disease}

In the four patients with $\mathrm{HbS} / \mathrm{C}$ disease, recurrent VOCs / APCs were observed in two patients. Both patients had a medical history of VOCs/APCs, however, none of them resulted in inpatient admission during pregnancy. One of the pregnancies was further complicated by transfusion demand for RBCs due to symptomatic anemia unrelated to a VOC/APC. Peripartum (SCD-associated) complications were observed in one of the pregnancies and required inpatient admission due to a VOC (pyelonephritis) in the third trimester of pregnancy which progressed to an ACS, requiring CS. AIS was seen in one of the four patients. In the postpartum period, only in one of the two patients with past VOCs / APCs during gestational phase were VOCs / APCs also documented, respectively. Throughout observation time, no serious infectious- or TE complications were observed.

Three of the overall seven successful pregnancies in the other two $\mathrm{HbS} / \mathrm{C}$ patients were uneventful $(43 \%, 3 / 7)$, despite documented VOCs/APCs prior to conception in one of them.

\subsection{Neonatal Outcomes}

In patients with severe genotypes, median GW at delivery was 37 weeks (range 29-40; $\mathrm{N}=24$ ) compared to 38 weeks (range, 37-40; $\mathrm{N}=6 / 7$ ) in $\mathrm{HbS} / \mathrm{C}$ patients. Preterm neonates defined as $<37$ weeks of completed gestational age, were observed in $42 \%(10 / 24)$ of deliveries in patients with severe genotypes. In comparison, the rate of preterm neonates from mothers with $\mathrm{HbS} / \mathrm{C}$ disease for whom gestational age was available was $14 \%(1 / 7)$. Median overall birth weight of neonates of mothers with severe 
disease-associated genotypes was $2570 \mathrm{~g}$ (range, 946-3090 g; $\mathrm{N}=19$ ) (median (overall) height: $47 \mathrm{~cm}$ (range, 36-50; $\mathrm{N}=17$ )); median (overall) head circumference: $32 \mathrm{~cm}$ (range, 27.2-34.5; $\mathrm{N}=17)$.

Excluding preterm neonates $(\mathrm{N}=8)$, data of neonatal outcomes were available from 12 neonates of HbSS patients. Median birth weight in these neonates was $2677 \mathrm{~g}$ (range, 1980-2090); height (median): $47.5 \mathrm{~cm}$ (range, 46-50); and head circumference (median): $32 \mathrm{~cm}$ (range, 30-34.5; $\mathrm{N}=21$ )). Neonates from mothers with $\mathrm{HbS} / \mathrm{C}$ disease born >37 GW displayed a median birth weight of $3370 \mathrm{~g}$ (range, 2890-4330), a height (median) of $55 \mathrm{~cm}$ (range, 51-60); and head circumference (median) of $35 \mathrm{~cm}$ (range, 34-36.5). Thus, there were statistically significant differences with respect to neonatal growth in favor of neonates from $\mathrm{HbS} / \mathrm{C}$ mothers (Figure 1). A preterm CS (GW 29+5) in one patient with $\mathrm{HbSS}-\alpha^{+}$-thalassemia and platelet delta-storage pool disease was required in the context of right-sided subdural fetal hematoma $(1.5 \times 7 \mathrm{~cm})$ with midline shift and ventricular enlargement of the lateral ventricles.
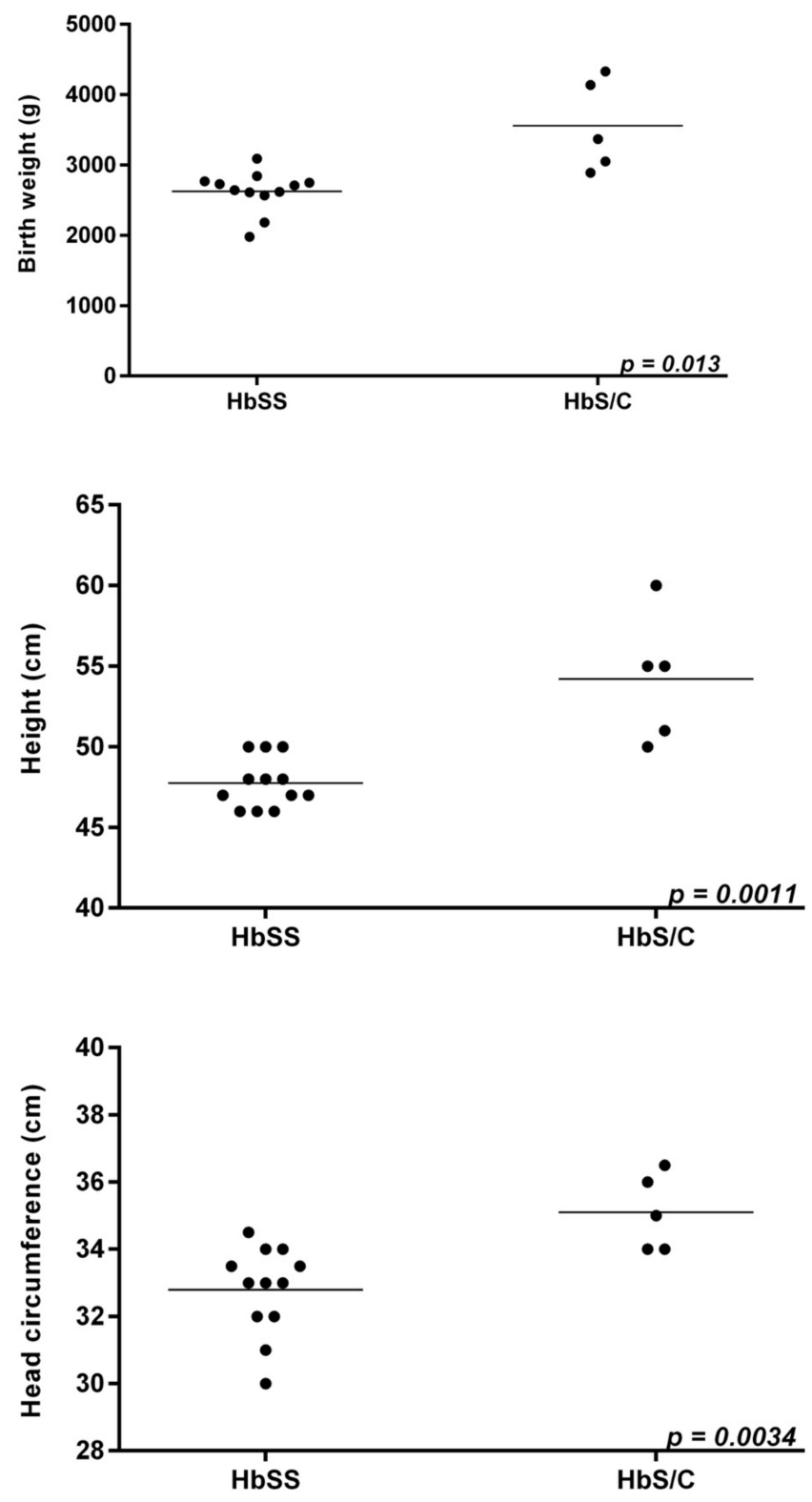

Figure 1. Neonatal status (Birth weight $(\mathrm{g})$, height $(\mathrm{cm})$, and head circumference $(\mathrm{cm})$ ) from women with HbSS $(\mathrm{N}=12)$ versus HbS/C disease $(\mathrm{N}=5) \pm$ co-inheritance of heterozygous $\alpha$-thalassemia, excluding preterm neonates. 


\section{Discussion}

Pregnancy in SCD is associated with a high incidence of adverse events as highlighted by past meta-analyses implicating the importance of an interdisciplinary therapeutic approach by national reference centers to ensure maternal and fetal/neonatal well-being $[4,12]$. The results of our single-center study are in partial agreement with previous studies.

Before commenting on the findings in our patients, we have to point out on the low number of patients with severe genotypes receiving $\mathrm{HC}$, given the overall benefit regarding reduction in morbidity and mortality rates in HC-treated SCD patients [13-15]. The low number was based on the following notion: some of the patients rejected treatment because of individual concerns. Others were not taken care by a medical reference center prior to pregnancy and, thus, had only very limited access to antenatal care. These patients presented at our department at a late stage of pregnancy which may be attributed to their refugee status, often requiring direct hospitalization due to SCD-associated complications. Furthermore, despite the rising prevalence of SCD in Germany due to recent immigration, SCD is still considered a rare disease compared to other countries. Nevertheless, as the present analysis summarizes outcomes over a period of two decades, our results support the findings of other studies stating an overall improved maternal and neonatal outcome over the past decades $[3,16,17]$.

In a meta-analysis published by Oteng-Ntim et al. (2015), the authors estimated a sixfold increased risk of maternal mortality in HbSS females compared to non-affected persons [4]. However, mortality rates vary depending on the particular analyses, the time of data collection, and may likely be associated with a poorer health care system as compared to Europe. Even though, no increased maternal mortality is observed in developing countries with substantial experience, as published by Babah et al. (2019) [18].

Although a low prevalence of HC-induced teratogenicity can be assumed following exposure during pregnancy, treatment is supposed to be withheld three months before conception, if feasible [19]. Of note, in all our documented pregnancies (except one) with prior maternal HC exposure (treatment duration: $\geq 1$ year), treatment was stopped only after diagnosis of pregnancy within the 1st trimester, indicating that all fetuses were exposed to HC during organogenesis (3rd to 8th week after conception). In these patients no obvious difference with regard to fetal mortality was observed (i.e., spontaneous abortions compared to HC-naive mothers) and neonatal development was not associated with any adverse events throughout observation time. Furthermore, in one of our patients with known alloantibodies and severe transfusion-dependent anemia, HC was needed to be reinitiated in the 3rd trimester of pregnancy up to four weeks prior to delivery during her two consecutive pregnancies resulting in no adverse fetal outcome. Our observations are therefore in line with the results observed in the European non-interventional, multicentric, prospective Escort-HU study. Hence, in some patients not suitable for SP(E)BT, HC $( \pm E P O)$ may remain the only therapeutic option to ensure maternal and fetal well-being. Noteworthy, HC might even be considered safe in some patients if treated throughout the entire pregnancy. However, decision should be based on a case-by-case discussion and cannot be generally recommended due to the potential harmful effects [20].

Whether or not SPBTs are beneficial in pregnant SCD patients remains a matter of debate [21-24]. Importantly, transfusion techniques and timing of SP(E)BT vary throughout the different studies probably explaining the contradictory results. Our study was not designed to answer this important topic. However, we did observe a high demand for RBC transfusions, especially in HbSS patients. Taking the risk of alloimmunization into account, transfusions were restricted to patients presenting either with symptomatic/hemolytic anemia or to those with other SCD-associated or obstetric complications.

Alloimmunization is a multifactorial process and presents one of the most clinically relevant complications in the context of pregnancy. The incidence of alloimmunization is highest in SCD patients, with a reported rate of up to 47\% [25]. Most of the alloantibodies are formed against Rhesus- (Rh-) and Kell- (K-) antigens [1,26]. It is assumed that this 
is due to the much larger variety of Rh-antigens found outside of Europe, particularly Africa, which differ from the common variants of European blood donors [1]. We observed alloimmunization in two out of eight HbSS patients with a history of periodic blood transfusions. Our low rate may be related to extended red cell antigen typing which may lower the rate of alloimmunization, although this has not yet been clearly proven [27]. Nevertheless, even in patients with a history of low exposure to allogeneic RBCs, alloimmunization can still occur at a relatively high rate, as reported for Ugandan sickle cell patients (with $6.1 \%$ alloimmunization) [28]. Although extended RBC matching has not been formally proven to contribute to a reduction of alloimmunization rates, it can speed up identification of the specificity of alloantibodies in the case of a positive antibody screen and also helps to select compatible RBCs [27]. Furthermore, some clinically significant alloantibodies can be difficult to detect because they evanesce over time and drop below the detection threshold in the immunohematology laboratory [29]. This potentiates the risk for severe transfusion-associated complications after antigen-re-exposure by transfusion of antigen-positive red blood cells. Such a booster can particularly lead to DHTRs [30]. It also emphasizes the importance of extended RBC antigen characterization (serologically and/or genetically) of donors and recipients, especially in European countries with a low disease prevalence to decrease the risk of potential transfusion reactions [31]. Particularly intrauterine transfusions can efficiently trigger alloimmunization against RBC antigens and against HLA molecules. As a matter of fact, alloimmunization against additional RBC antigens has been reported to occur in up to $25 \%$ of women already expressing an alloantibody. In contrast to transfusions in adults, extended antigen matching of donor cells (including Duffy-, Kidd-, and S-antigens) prior to intrauterine transfusion was shown to decrease the formation of alloantibodies in the mothers by $60 \%[32,33]$.

In summary, pregnant mothers suffering from SCD who have experienced severe SCD-related complications, either preceding the current pregnancy or during a previous pregnancy, may be considered for SP(E)BTs. This is the case if a high-risk exists for both, the mother and the fetus (e.g., twin pregnancies), or in patients encountering SCD-associated complications during the current pregnancy, in patients receiving $\mathrm{HC}$ before the gravidity, or in patients already receiving a chronic transfusion program [27]. A switch to SP(E)BTs may be worth to be considered in patients receiving crizanlizumab prior to or in the context of pregnancy for prevention of recurrent VOCs. Whether outcome might be favored by early initiation of SPEBT as described by Vianello et al. (2018) may be answered by the TAPS-2 study, as placental damage in SCD might even occur early in pregnancy [34,35].

Because not all documented pregnancies were monitored at our department, we included a patient survey. Pregnancies in our patients with successful outcomes were accompanied by a high rate of vaso-occlusive events (VOEs), especially in HbSS patients $(96 \%)$ compared to $\mathrm{HbS} / \mathrm{C}$ disease, requiring in part, if severe, recurrent inpatient admissions for medical care. Of note, the patient query revealed that not all of them seeked advice of their treating physicians during a painful crisis. This may offer an explanation for the overall higher VOC/APC rates described in our patients unlike other studies. Nevertheless, routine counselling was associated with a sense of security according to the patient survey, as these patients could directly contact their attending physician in the event of impending complications, if desired.

Complicated VOEs (ACS) requiring inpatient admission for partial RCE and intravenous anti-infective treatment were observed for four pregnancies $(13 \%, 4 / 31)$, including a patient with $\mathrm{HbS} / \mathrm{C}$ disease. Thus, patients with $\mathrm{HbS} / \mathrm{C}$ disease also are at increased risk for serious complications when being pregnant or in the postpartum period. Nevertheless, a higher rate of complications was seen in patients with genotypes mediating severe disease.

Special attention should also be paid to the high number of elective abortions in our study. Reasons for medical abortions were related to age at time of conception, socioeconomic factors, including uncertain partnerships, or disease-related stressors, implicating the importance of early psycho-social support in patients with SCD. 
Author Contributions: Conceptualization, F.A.; Data curation, F.A.; Formal analysis, P.P. and F.A.; Investigation, P.P., R.Y. and F.A.; Methodology, R.Y. and F.A.; Project administration, F.A.; Supervision, F.A.; Validation, P.P., L.D., C.A.-S., N.K., A.I., M.P., C.T., H.K., V.L., M.K., A.C., H.C.R., A.K., A.R., R.Y., U.D. and F.A.; Visualization, F.A.; Writing—original draft, P.P. and F.A.; Writing—review \& editing, H.K., H.C.R., A.K., A.R., U.D. and F.A. All authors have read and agreed to the published version of the manuscript.

Funding: FA was supported as a Clinician Scientist within the University Medicine Essen Academy (UMEA) program, funded by the German Research Foundation (DFG; grant FU356/12-1) and the Faculty of Medicine, University of Duisburg-Essen, Germany.

Institutional Review Board Statement: The study involving human participants was reviewed and approved by retrospective analysis and use of data was approved by the Ethical Committee of the Faculty of Medicine at the University Hospital of Duisburg-Essen (21-10031-BO; 5/6/2021) and the study was conducted in accordance to the Declaration of Helsinki.

Informed Consent Statement: The patients/participants provided their written informed consent to participate in this study.

Data Availability Statement: All datasets generated for this study are included in the article.

Acknowledgments: We thank all our patients for participation in this study.

Conflicts of Interest: All authors declare that the research was conducted in the absence of any commercial or financial relationships that could be construed as a potential conflict of interest.

\section{References}

1. Vichinsky, E.P.; Earles, A.; Johnson, R.A.; Hoag, M.S.; Williams, A.; Lubin, B. Alloimmunization in Sickle Cell Anemia and Transfusion of Racially Unmatched Blood. N. Engl. J. Med. 1990, 322, 1617-1621. [CrossRef]

2. Piel, F.B.; Steinberg, M.H.; Rees, D.C. Sickle Cell Disease. N. Engl. J. Med. 2017, 376, 1561-1573. [CrossRef]

3. Smith, J.; Espeland, M.; Bellevue, R.; Bonds, D.; Brown, A.; Koshy, M. Pregnancy in Sickle Cell Disease: Experience of the Cooperative study of sickle cell disease. Obstet. Gynecol. 1996, 87, 199-204. [CrossRef]

4. $\quad$ Oteng-Ntim, E.; Meeks, D.; Seed, P.T.; Webster, L.; Howard, J.; Doyle, P.; Chappell, L.C. Adverse maternal and perinatal outcomes in pregnant women with sickle cell disease: Systematic review and meta-analysis. Blood 2015, 125, 3316-3325. [CrossRef]

5. EU/3/16/1769 I European Medicines Agency. Available online: https://www.ema.europa.eu/en/medicines/human/orphandesignations/eu3161769 (accessed on 29 June 2021).

6. Adakveo I European Medicines Agency. Available online: https://www.ema.europa.eu/en/medicines/human/EPAR/adakveo (accessed on 29 June 2021).

7. Ballas, S.K. The Evolving Pharmacotherapeutic Landscape for the Treatment of Sickle Cell Disease. Mediterr. J. Hematol. Infect. Dis. 2020, 12, e2020010. [CrossRef] [PubMed]

8. Smith-Whitley, K. Complications in pregnant women with sickle cell disease. Hematology 2019, 1, 359-366. [CrossRef] [PubMed]

9. Lobitz, S. Perspektive: Neugeborenen -screening auf Sichelzellkrankheiten in Deutschland. Kinder Jugendmed. 2017, 17, 82-86. [CrossRef]

10. Wong, T.E.; Brandow, A.M.; Lim, W.; Lottenberg, R. Update on the use of hydroxyurea therapy in sickle cell disease. Blood 2014, 124, 3850-3857. [CrossRef]

11. Siklos I European Medicines Agency. Available online: https://www.ema.europa.eu/en/medicines/human/EPAR/siklos (accessed on 29 June 2021).

12. Boafor, T.K.; Olayemi, E.; Galadanci, N.; Hayfron-Benjamin, C.; Dei-Adomakoh, Y.; Segbefia, C.; Kassim, A.A.; Aliyu, M.H.; Galadanci, H.; Tuuli, M.G.; et al. Pregnancy outcomes in women with sickle-cell disease in low and high income countries: A systematic review and meta-analysis. BJOG Int. J. Obstet. Gynaecol. 2016, 123, 691-698. [CrossRef]

13. Bakanay, S.M.; Dainer, E.; Clair, B.; Adekile, A.; Daitch, L.; Wells, L.; Holley, L.; Smith, D.; Kutlar, A. Mortality in sickle cell patients on hydroxyurea therapy. Blood 2005, 105, 545-547. [CrossRef]

14. Ballas, S.K.; Barton, F.B.; Waclawiw, M.A.; Swerdlow, P.; Eckman, J.R.; Pegelow, C.H.; Koshy, M.; Barton, B.A.; Bonds, D.R. Hydroxyurea and sickle cell anemia: Effect on quality of life. Health Qual. Life Outcomes 2006, 4, 59. [CrossRef]

15. Voskaridou, E.; Christoulas, D.; Bilalis, A.; Plata, E.; Varvagiannis, K.; Stamatopoulos, G.; Sinopoulou, K.; Balassopoulou, A.; Loukopoulos, D.; Terpos, E. The effect of prolonged administration of hydroxyurea on morbidity and mortality in adult patients with sickle cell syndromes: Results of a 17-year, single-center trial (LaSHS). Blood 2010, 115, 12-2354. [CrossRef]

16. Jain, D.; Atmapoojya, P.; Colah, R.; Lodha, P. Sickle cell disease and Pregnancy. Mediterr. J. Hematol. Infect. Dis. 2019, 11, 2019040. [CrossRef] [PubMed]

17. Sun, P.M.; Wilburn, W.; Raynor, B.; Jamieson, D. Sickle cell disease in pregnancy: Twenty years of experience at Grady Memorial Hospital, Atlanta, Georgia. Am. J. Obstet. Gynecol. 2001, 184, 6-1127. [CrossRef] 
18. Babah, O.A.; Aderolu, M.B.; Oluwole, A.A.; Afolabi, B.B. Towards zero mortality in sickle cell pregnancy: A prospective study comparing haemoglobin SS and AA women in Lagos, Nigeria. Niger. Postgrad. Med. J. 2019, 26, 1-7. [CrossRef]

19. Gellen-Dautremer, J.; Le Jeune, S.; Receveur, M.-C.; Foïs, E. Hydroxyurea Exposure throughout Pregnancy in Patients with Sickle-Cell Disease: 4 Case Reports from European Non-Interventional, Multicentric, Prospective Escort-HU Study. Blood 2019, 134, 1027. [CrossRef]

20. Galactéros, F.; Cannas, G.; Bartolucci, P.; Voskaridou, E.; Joseph, L.; Bernit, E.; Gellen-Dautremer, J.; Charneau, C.; Ngo, S.; Habibi, A. Outcomes of Pregnancies in Patients with Sickle-Cell Disease: Update from European Non-Interventional, Multicentric, Prospective Escort-HU Study. Blood 2019, 134, 891. [CrossRef]

21. Malinowski, A.K.; Shehata, N.; D'Souza, R.; Kuo, K.H.M.; Ward, R.; Shah, P.S.; Murphy, K. Prophylactic transfusion for pregnant women with sickle cell disease: A systematic review and meta-analysis. Blood 2015, 126, 2424-2435. [CrossRef]

22. Elenga, N.; Adeline, A.; Balcaen, J.; Vaz, T.; Calvez, M.; Terraz, A.; Accrombessi, L.; Carles, G. Pregnancy in Sickle Cell Disease Is a Very High-Risk Situation: An Observational Study. Obstet. Gynecol. Int. 2016, 2016, 9069054. [CrossRef]

23. Whittington, J.R.; Magann, E.F.; Ounpraseuth, S.T.; Chang, J.N.; Whitcombe, D.D.; Morrison, J.C. Evidence for Prophylactic Transfusion during Pregnancy for Women with Sickle Cell Disease. South. Med. J. 2021, 114, 231-236. [CrossRef] [PubMed]

24. Driss, F.; Tertian, G.; Becquemont, L.; Haddad, B.; Cynober, T.; Raphael, M.; Tchernia, G. Prise en charge des grossesses à risque chez les femmes drépanocytaires: Intérêt d'une stratégie préventive par des transfusions de globules rouges ou des échanges érythrocytaires automatisés. Transfus. Clin. Biol. 2007, 14, 386-392. [CrossRef]

25. Chou, S.T.; Jackson, T.; Vege, S.; Smith-Whitley, K.; Friedman, D.F.; Westhoff, C.M. High prevalence of red blood cell alloimmunization in sickle cell disease despite transfusion from Rh-matched minority donors. Blood 2013, 122, 1062-1071. [CrossRef] [PubMed]

26. Sippert, E.; Fujita, C.R. Variant RH alleles and Rh immunisation in patients with sickle cell disease. Blood Transfus. 2015, 13, 72. [CrossRef]

27. Chou, S.T.; Alsawas, M.; Fasano, R.M.; Field, J.J.; Hendrickson, J.E.; Howard, J.; Kameka, M.; Kwiatkowski, J.L.; Pirenne, F.; Shi, P.A.; et al. American Society of Hematology 2020 guidelines for sickle cell disease: Transfusion support. Blood Adv. 2020, 28, 327-355. [CrossRef] [PubMed]

28. Natukunda, B.; Schonewille, H.; Ndugwa, C.; Brand, A. Red blood cell alloimmunization in sickle cell disease patients in Uganda. Transfusion 2010, 50, 20-25. [CrossRef]

29. Tormey, C.A.; Stack, G. The persistence and evanescence of blood group alloantibodies in men. Transfusion 2009, 49, 505-512. [CrossRef] [PubMed]

30. Nickel, R.S.; Hendrickson, J.E.; Fasano, R.M.; Meyer, E.K.; Winkler, A.M.; Yee, M.M.; Lane, P.A.; Jones, Y.A.; Pashankar, F.D.; New, T.; et al. Impact of red blood cell alloimmunization on sickle cell disease mortality: A case series. Transfusion 2016, 56, 107-114. [CrossRef]

31. Costa, D.C.D.; Pellegrino, J., Jr.; Guelsin, G.A.S.; Ribeiro, K.A.R.; Gilli, S.C.O.; Castilho, L. Molecular matching of red blood cells is superior to serological matching in sickle cell disease patients. Rev. Bras. Hematol. Hemoter. 2013, 35, 35-38. [CrossRef]

32. Schonewille, H.; Prinsen-Zander, K.J.; Reijnart, M.; Van De Watering, L.; Zwaginga, J.-J.; Meerman, R.H.; Van Kamp, I.L.; Brand, A. Extended matched intrauterine transfusions reduce maternal Duffy, Kidd, and S antibody formation. Transfusion 2015, 55, 2912-2919. [CrossRef]

33. Hendrickson, J.E.; Tormey, C.A. Understanding red blood cell alloimmunization triggers. Hematology 2016, $2016,446-451$. [CrossRef] [PubMed]

34. Oakley, L.L.; Awogbade, M.; Brien, S.; Briley, A.; Chorozoglou, M.; Drasar, E.; Johns, J.; Rhodes, E.; Robinson, V.; Seed, P.; et al. Serial prophylactic exchange blood transfusion in pregnant women with sickle cell disease (TAPS-2): Study protocol for a randomised controlled feasibility trial. Trials 2020, 21, 1-11. [CrossRef] [PubMed]

35. Vianello, A.; Vencato, E.; Cantini, M.; Zanconato, G.; Manfrin, E.; Zamo, A.; Zorzi, F.; Mazzi, F.; Martinelli, N.; Cavaliere, E.; et al. Improvement of maternal and fetal outcomes in women with sickle cell disease treated with early prophylactic erythrocytapheresis. Transfusion 2018, 58, 2192-2201. [CrossRef] [PubMed] 